\title{
Biological and chemical forms in controlling oral biofilms
}

\section{Samar A. Bamofleh ${ }^{1 *}$, Mohammed S. Almuammar², Aljawhara I. Bin Osseil ${ }^{2}$, Roaa A. Alorf ${ }^{3}$, Sultan M. Alqhtani ${ }^{4}$, Amal A. Alsadah ${ }^{5}$, Sarah M. Abdullah ${ }^{6}$, Donya E. Ganboa ${ }^{7}$, Basil F. Abualkhair ${ }^{8}$, Mohammed A. Khojah', Bashaer M. Alhawsawi ${ }^{10}$}

\author{
${ }^{1}$ North Jeddah Specialist Dental Center, King Abdullah Medical Complex, Jeddah, Saudi Arabia \\ ${ }^{2}$ College of Dentistry, King Saud University, Riyadh, Saudi Arabia \\ ${ }^{3}$ College of Dentistry, King Saud bin Abdulaziz University for Health Sciences, Riyadh, Saudi Arabia \\ ${ }^{4}$ College of Dentistry, Taif University, Taif, Saudi Arabia \\ ${ }^{5}$ General Dentist, Ministry of Health, Eastern Region, Saudi Arabia \\ ${ }^{6}$ Dental Department, Shaqra General Hospital, Shaqra, Saudi Arabia \\ ${ }^{7}$ General Dentist, Khalid Advanced Center for General Medicine, Jeddah, Saudi Arabia \\ ${ }^{8}$ College of Dentistry, Taibah University, Medina, Saudi Arabia \\ ${ }^{9}$ King Abdulaziz Primary Healthcare Center, King Abdulaziz University Hospital, Jeddah, Saudi Arabia \\ ${ }^{10}$ General Dentist, Ministry of Health, Medina, Saudi Arabia
}

Received: 14 December 2021

Accepted: 29 December 2021

\section{*Correspondence:}

Dr. Samar A. Bamofleh,

E-mail: samarabamofleh@gmail.com

Copyright: (C) the author(s), publisher and licensee Medip Academy. This is an open-access article distributed under the terms of the Creative Commons Attribution Non-Commercial License, which permits unrestricted non-commercial use, distribution, and reproduction in any medium, provided the original work is properly cited.

\begin{abstract}
Although there have been many advances in the medical field regarding disease control and management, it has been demonstrated that certain diseases and infections still represent a significant challenge. For example, the presence of oral biofilms indicates the virulence of the underlying infection in different dental infections diseases, including periimplantitis, periapical periodontitis, periodontitis, gingivitis, and dental caries. We have discussed various mechanical, chemical, and biological modalities that can be applied to control biofilms and limit plaque formation and secondary caries. Although physical brushing might be efficacious in cleaning, evidence indicates that it cannot eradicate the underlying bacteria. Accordingly, using biological and chemical materials is essential to achieve adequate disinfection and enhance the outcomes. Many modalities have been proposed in the literature, such as nanomaterials, organic compounds such as arginine, dietary substances, and the various chemical oral cleansers discussed in the current study. Bacteriophages are also promising in this context. However, they need further exploration regarding their efficacy and safety. In addition, resistance against these compounds is a serious issue and needs to be addressed in future research.
\end{abstract}

Keywords: Cleansers, Dental plaques, Biofilms, Treatment, Endodontics, Biological, Chemical

\section{INTRODUCTION}

The presence of oral biofilms indicates the virulence of the underlying infection in different dental infections diseases, including peri-implantitis, periapical periodontitis, periodontitis, gingivitis, and dental caries. ${ }^{1-5}$ Estimates show that the process of controlling these biofilms is associated with a major economic burden worldwide. ${ }^{6}$ However, recent medical advances enhanced the efficacy of the relevant modalities to reduce the burdens of biofilms and enhance their control. ${ }^{7,8}$ This has been associated with enhanced therapeutic outcomes and better disease control.

Although there have been many advances in the medical field regarding disease control and management, it has been demonstrated that certain diseases and infections still represent a major challenge. For example, some infections can accumulate on medical devices and lead to biofilm 
formation, which might worsen the treatment outcomes and even intervene against these medical devices' functions. Many relevant organisms were reported as the main source for these events in this context. These include Mycobacteria, Streptococcus, Listeria, Salmonella, Escherichia, Virbrio, and Pseudomonas. ${ }^{9}$ Fortunately, evidence indicates that certain biological, mechanical, and chemical approaches can control these biofilms. The present literature review will discuss the efficacy of different biological and chemical approaches in controlling oral biofilms based on data from relevant studies.

\section{METHODS}

This literature review is based on an extensive literature search in Medline, Cochrane, and EMBASE databases on which was performed 03 December 2021 using the medical subject headings $(\mathrm{MeSH})$ or a combination of all possible related terms, according to the database. To avoid missing poetential studies, a further manual search for papers was done through Google Scholar, while the reference lists of the initially included papers. Studies discussing biological and chemical forms in controlling oral biofilms were screened for useful information, with no limitations posed on date, language, age of participants, or publication type.

\section{DISCUSSION}

It is widely known that the process of controlling biofilms is challenging. However, evidence indicates that many strategies exist to control these modalities adequately. Among the different methods that have been reported in the literature, these might include physical and mechanical approaches, like brushing. The present section will discuss all reported approaches, including biological and chemical methods. For example, studies show that physically removing biofilms from dentures can be easily conducted with favorable outcomes to prevent the spread and development of infection. However, other opinions indicate that physical removal alone might not be sufficient to produce favorable outcomes. Therefore, biological and chemical materials should be used to achieve more enhanced outcomes and adequate control of biofilms. ${ }^{10,11}$

Among the different approaches, evidence shows that the administration of alkaline peroxides can effectively lead to enhanced cleaning outcomes. It is usually used in the form of tablets, which produce an effervescent alkaline solution when added to water, releasing active oxygen and hydrogen peroxide. $^{12}$ The released materials can effectively clean and eliminate regions where brushing cannot be effectively used. In this context, a previous relevant investigation aimed to study the efficacy of immersing dentures in dental cleansers daily to find the outcomes on controlling biofilms. The authors reported that they immersed acrylic resin dentures, upon which multispecies biofilms were cultivated, into a commercially available alkaline peroxide enzyme-containing denture cleanser in a daily laboratory-based pattern for three minutes per immersion. Furthermore, the authors reported conducting this approach for seven consecutive days and compared the findings with a control group. Moreover, it has been shown that the parameters used to quantify biofilm control and evaluate the results include extracellular polysaccharide formation and colonyforming units. Finally, it has been concluded that daily immersion of dentures into these cleansers was significantly associated with favorable outcomes, as the total number of organisms was significantly reduced on the denture surface. Besides, it has been shown that they managed to achieve complete elimination of certain species, including $V$. dispar and $F$. nucleatum, following a day of immersion. ${ }^{13}$

Other investigations also compared the cleaning efficacies of chemical (effervescent alkaline) and mechanical (brushing with dentifrice) hygiene methods. For instance, Paranhos et al demonstrated that both mechanical and chemical approaches are equally effective in eradicating certain bacterial biofilms but not for others. ${ }^{14}$ For instance, the authors reported that both modalities were equally effective in eradicating $P$. aeruginosa, S. mutans, and $S$. aureus. On the other hand, mechanical approaches (either alone or in combination with the chemical ones) eradicated C. glabrata, C. Albicans, and E. faecalis more significantly than when the chemical methods were used alone. ${ }^{15}$

Other studies also proved the efficacy and effectiveness of antiplaque oral rinses in eradicating biofilms. Although the efficacy of these modalities is well-established in eradicating plaques, it has been shown that some side effects might be associated with the application of compounds that contain essential oils and others that contain chlorhexidine gluconate. For instance, it has been shown that occasional loss of taste and tooth discoloration might be encountered following the administration of chlorhexidine gluconate. On the other hand, evidence indicates that using compounds that contain essential oils is preferred because these modalities usually have reduced frequencies of adverse events as they represent reduced mammalian toxicities. Furthermore, some authors suggested that adding certain enzymes to these oral formulations might enhance their cleansing efficacy. ${ }^{16}$ In another context, some studies also validated the efficacy of using natural herbal-based mouth cleansers in eradicating infections and controlling biofilms. For instance, it has been shown that a mouth rinse, which is alcohol-free, commercially available, and herbal-based (that contains Sambucus nigra, Echinacea purpurea, and Centella Asiatica), is effective in controlling biofilms. In addition, a previous study reported that the compound is significantly effective against eliminating and reducing the frequency of dental plaque build-up based on its components' natural antimicrobial and anti-inflammatory features. ${ }^{17}$ Moreover, the efficacy of these modalities has been furtherly elaborated among patients that were asked to use these herbal-based modalities with disposing of other oral rinsers for two weeks. The authors reported that the efficacy of herbal-based cleansers was superior to the control approaches in preventing gingival inflammation 
and plaque formation. ${ }^{17}$ Although the efficacy of these approaches is well-established in the literature, many efforts are still being reported for developing more efficacious therapeutic modalities with better control over biofilms to enhance treatment outcomes. ${ }^{18,19}$

It should also be noted that many specific nanomaterials have been validated as efficacious approaches for managing and controlling dental biofilms. For instance, it has been indicated that silver nanoparticles and silver nitrates are the most efficacious modalities to achieve adequate control of dental biofilms. ${ }^{20}$ However, some studies reported that these materials could be associated with dental discoloration. ${ }^{1,21,22}$ On the other hand, many studies reported that direct dentin silver nanocoating could successfully achieve adequate control of biofilms and bacterial growth inhibition. Accordingly, these approaches can protect against secondary caries and dental plaques when applied over dentin. ${ }^{1}$ Moreover, some studies also reported that nanomaterials are significantly efficacious in eradicating Enterococcus faecalis, which has been associated with the development of persistent and secondary dental infections. ${ }^{23,24}$ Furthermore, another study reported that the efficacy of these modalities could be further increased when added to calcium hydroxide, which has been reported to be highly effective as a shortterm disinfectant that might help control biofilms. ${ }^{25}$ However, it should be noted that the application approaches of nanomaterials as silver nanoparticles might impair their efficacies against biofilms. ${ }^{26,27}$

Quaternary ammonium salts (QAS) were also reported in the literature as efficacious approaches that have been used to control biofilms since the 1970 s. ${ }^{28,29}$ Many categories of this modality have been proposed as efficacious modalities that can intervene against bacterial infections and dental plaque formation. For instance, a previous study reported that 12-methacryloyloxydodecyl-pyridinium bromide (MDPB) is significantly effective against many bacterial infections pathogens as $F$. nucleatum, E. Faecalis, $S$. mutans, and Prevotella nigrescens. ${ }^{30,31}$ In addition, evidence shows that these modalities are highly efficacious in inhibiting adherence and the growth of bacteria. Recent evidence furtherly indicates that novel antibacterial monomers dimethylaminododecyl methacrylate (DMADDM) and dimethylaminohexadecyl methacrylate (DMAHDM) can enhance the antibacterial activities of quaternary ammonium dimethacrylate (QADM), an efficacious type of QAS. ${ }^{32,33}$ In this context, a previous in vitro investigation reported that the complete eradication of S. mutans was significantly associated with applying $10 \%$ DMAHDM of bonding agent. ${ }^{34}$ Besides, different combinations of QAS and their types were also proposed in the literature were favorable outcomes and antibacterial activities that might reduce bacterial overgrowth and plaque formation. ${ }^{35}$ However, it should be noted that the bacterial resistance might develop secondary to the frequent administration of QAS. ${ }^{36}$ For instance, the resistance of four bacterial species (including $P$. gingivalis, F. nucleatum, E. faecalis, and Streptococcus gordonii) has been reported against chlorhexidine. ${ }^{37}$ On the other hand, other studies reported that no resistance from E. faecalis or S. mutans was noticed with the application of MDPB. ${ }^{38}$ Another study reported that resistance was also noticed against DMAHDM and DMADDM from eight bacterial species (including Prevotella intermedia, $P$. gingivalis, $S$. nucleatum, A. actinomycetemcomitans, E. faecalis, $S$. gordonii, S. sanguinis, and S. mutans).$^{37}$

Disrupting the formation of biofilms was also reported as another efficacious approach to control it. This has been reported to be effectively induced by applying small molecules. The literature also reported using arginine as an effective antimicrobial modality that can control biofilms and enhance treatment efficacy with no adverse events. The efficacy of arginine has been reported against different bacterial pathogens. In the same context, previous studies reported that various natural products could serve as natural cleansers against many bacterial with no need for chemical or mechanical substances and materials. For instance, many investigations in the literature reported tea, propolis, and cranberry with favorable effects and reduced adverse events compared to the aforementioned bactericidal modalities (Figure 1). ${ }^{15}$
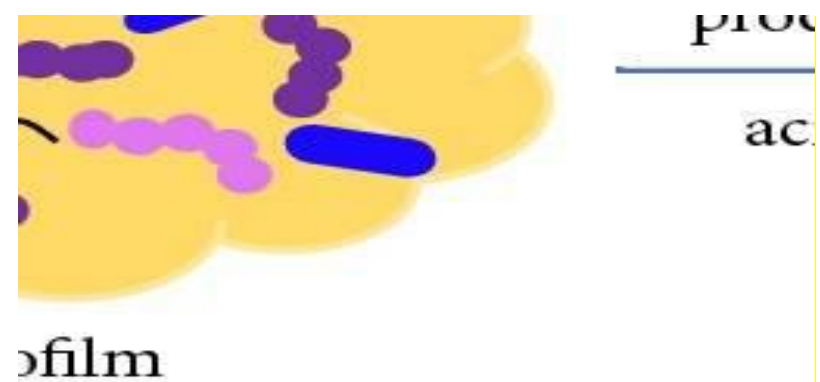

Figure 1: The bactericidal effects of various materials against $S$. mutans and dental plaque formation. ${ }^{15}$

Some studies also reported that some modalities could be used as promising approaches that can be used to control biofilms. However, these modalities are still under investigation and need further application and wide analysis for efficacy and safety. For instance, bacterial viruses or bacteriophages can be effectively used as effective bactericidal approaches with minimal adverse events. These modalities were reported to disrupt bacterial growth and eradicate them, including E. coli biofilms. ${ }^{17}$ In addition to the minimal adverse events reported with these modalities, it has been furtherly reported that these approaches are comparatively cheap and easily reproducible compared with other materials. However, it should be noted that the application of these materials on human subjects is still under investigation. ${ }^{39}$ A previous study by Eckert et al also reported that specifically- 
targeted AMPs (STAMPs) could be used as efficacious modalities that can be used to eradicate certain bacteria and other pathogens and prevent dental plaque formation. ${ }^{40}$ The authors reported that these modalities are significantly efficacious against $S$. mutans. However, it has been furtherly reported that these modalities did not have any favorable effects against other bacterial pathogens, including S. sanguinis and S. Gordonii. ${ }^{40}$ Finally, it has been shown that these modalities also have several advantages, being colorless, tasteless, odorless, and having no staining impacts, which makes them superior candidates against certain bacterial pathogens. However, further research is still needed to target their efficacy against other bacteria. Another adjuvant therapy modality that can enhance the efficacy of other bactericidal approaches is quorum sensing (QS) inhibition, which has been discovered in many pathogens, including Streptococcus, E. coli, Pseudomonas, V. fischeri, and even fungi as Candida Albicans. However, it should be noted that the efficacy of these modalities is still under investigation in different ongoing research studies. ${ }^{41,42}$ Finally, these modalities can only be used as adjuvant modalities that limit the spread of bacteria and other pathogens. However, they cannot eradicate them. Therefore, other primary disinfectants and cleansers are always needed with their administration. ${ }^{43,44}$

\section{CONCLUSION}

Although physical brushing might be efficacious in cleaning, evidence indicates that it cannot eradicate the underlying bacteria. Accordingly, using biological and chemical materials is essential to achieve adequate disinfection and enhance the outcomes. Many modalities have been proposed in the literature, such as nanomaterials, organic compounds such as arginine, dietary substances, and the various chemical oral cleansers discussed in the current study. Bacteriophages are also promising in this context. However, they need further exploration regarding their efficacy and safety. In addition, resistance against these compounds is a serious issue and needs to be addressed in future research.

\section{Funding: No funding sources Conflict of interest: None declared Ethical approval: Not required}

\section{REFERENCES}

1. Besinis A, De Peralta T, Handy RD. Inhibition of biofilm formation and antibacterial properties of a silver nano-coating on human dentine. Nanotoxicology. 2014;8(7):745-54.

2. Liljemark WF, Bloomquist C. Human oral microbial ecology and dental caries and periodontal diseases. Crit Rev Oral Biol Med. 1996;7(2):180-98.

3. Ricucci D, Siqueira JF, Jr. Biofilms and apical periodontitis: study of prevalence and association with clinical and histopathologic findings. J Endod. 2010;36(8):1277-88.
4. Davies D. Understanding biofilm resistance to antibacterial agents. Nature Rev Drug Discovery. 2003;2(2):114-22.

5. Noiri Y, Ehara A, Kawahara T, Takemura N, Ebisu S. Participation of bacterial biofilms in refractory and chronic periapical periodontitis. $\mathrm{J}$ Endod. 2002;28(10):679-83.

6. Beikler T, Flemmig TF. Oral biofilm-associated diseases: trends and implications for quality of life, systemic health and expenditures. Periodontology 2000. 2011;55(1):87-103.

7. Yang L, Liu Y, Wu H, Hóiby N, Molin S, Song ZJ. Current understanding of multi-species biofilms. Int J Oral Sci. 2011;3(2):74-81.

8. Flemming HC, Wingender J, Szewzyk U, Steinberg P, Rice SA, Kjelleberg S. Biofilms: an emergent form of bacterial life. Nat Rev Microbiol. 2016;14(9):563-75.

9. Hall-Stoodley L, Stoodley P. Developmental regulation of microbial biofilms. Curr Opinion Biotechnol. 2002;13(3):228-33.

10. Thieu H, Bach Dat B, Nam NH, et al. Antibiotic resistance of Helicobacter pylori infection in a children's hospital in Vietnam: prevalence and associated factors. Minerva medica. 2020;111(5):498-501.

11. Nguyen TM, Huan VT, Reda A, Morsy S, Nam Giang HT, Tri VD, et al. Clinical features and outcomes of neonatal dengue at the Children's Hospital 1, Ho Chi Minh, Vietnam. J Clin Virol. 2021;138:104758.

12. Berger D, Rakhamimova A, Pollack A, Loewy Z. Oral Biofilms: Development, Control, and Analysis. High-throughput. 2018;7(3).

13. Lucena-Ferreira SC, Ricomini-Filho AP, Silva WJ, Cury JA, Cury AA. Influence of daily immersion in denture cleanser on multispecies biofilm. Clin Oral Investig. 2014;18(9):2179-85.

14. Paranhos HF, Silva-Lovato CH, Souza RF, Cruz PC, Freitas KM, Peracini A. Effects of mechanical and chemical methods on denture biofilm accumulation. J Oral Rehabilitation. 2007;34(8):606-12.

15. Kuang X, Chen V, Xu X. Novel Approaches to the Control of Oral Microbial Biofilms. BioMed Res Int. 2018;6498932.

16. Cortelli SC, Costa FO, Rode Sde M, Haas AN, Andrade AK, Pannuti CM, Escobar EC, Almeida ER, Cortelli JR, Pedrazzi V. Mouthrinse recommendation for prosthodontic patients. Braz Oral Res. 2014;28.

17. Levine WZ, Samuels N, Bar Sheshet ME, Grbic JT. A novel treatment of gingival recession using a botanical topical gingival patch and mouthrinse. J Contemp Dent Pract. 2013;14(5):948-53.

18. Son PT, Reda A, Viet DC. Exchange transfusion in the management of critical pertussis in young infants: a case series. Vox sanguinis. 2021;116(9):976-82.

19. El-Qushayri AE, Ghozy S, Reda A, Kamel AMA, Abbas AS, Dmytriw AA. The impact of Parkinson's disease on manifestations and outcomes of Covid-19 patients: A systematic review and meta-analysis. Rev Med Virol. 2021;e2278. 
20. Besinis A, De Peralta T, Handy RD. The antibacterial effects of silver, titanium dioxide and silica dioxide nanoparticles compared to the dental disinfectant chlorhexidine on Streptococcus mutans using a suite of bioassays. Nanotoxicology. 2014;8(1):1-16.

21. Peng JJ, Botelho MG, Matinlinna JP. Silver compounds used in dentistry for caries management: a review. J Dentistry. 2012;40(7):531-41.

22. Kumar A, Kumar V, Singh J, Hooda A, Dutta S. Drug-induced discoloration of teeth: an updated review. Clin Pediatrics. 2012;51(2):181-5.

23. Siqueira JF, Rôças IN. Exploiting molecular methods to explore endodontic infections: Part 2--Redefining the endodontic microbiota. J Endod. 2005;31(7):488-98.

24. Stuart CH, Schwartz SA, Beeson TJ, Owatz CB. Enterococcus faecalis: its role in root canal treatment failure and current concepts in retreatment. J Endod. 2006;32(2):93-8

25. Afkhami F, Pourhashemi SJ, Sadegh M, Salehi Y, Fard MJ. Antibiofilm efficacy of silver nanoparticles as a vehicle for calcium hydroxide medicament against Enterococcus faecalis. J Dentistry. 2015;43(12):1573-9.

26. Rodrigues CT, de Andrade FB, de Vasconcelos L. Antibacterial properties of silver nanoparticles as a root canal irrigant against Enterococcus faecalis biofilm and infected dentinal tubules. Int Endod J. 2018;51(8):901-11.

27. Wu D, Fan W, Kishen A, Gutmann JL, Fan B. Evaluation of the antibacterial efficacy of silver nanoparticles against Enterococcus faecalis biofilm. J Endod. 2014;40(2):285-90.

28. Liang J, Li M, Ren B, et al. The anti-caries effects of dental adhesive resin influenced by the position of functional groups in quaternary ammonium monomers. Dental Materials. 2018;34(3):400-11.

29. Ciancio SG, Mather ML, Bunnell HL. Clinical evaluation of a quaternary ammonium-containing mouthrinse. J Periodontol. 1975;46(7):397-401.

30. Izutani N, Imazato S, Noiri Y, Ebisu S. Antibacterial effects of MDPB against anaerobes associated with endodontic infections. Int Endod J. 2010;43(8):637-45.

31. Kitagawa R, Kitagawa $H$, Izutani $N$, Hirose $N$, Hayashi M, Imazato S. Development of an antibacterial root canal filling system containing MDPB. J Dent Res. 2014;93(12):1277-82.

32. Chen $\mathrm{C}$, Cheng L, Weir MD. Primer containing dimethylaminododecyl methacrylate kills bacteria impregnated in human dentin blocks. Int J Oral Sci. 2016;8(4):239-45.

33. Cheng L, Weir MD, Zhang K, Arola DD, Zhou X, Xu HH. Dental primer and adhesive containing a new antibacterial quaternary ammonium monomer dimethylaminododecyl methacrylate. J Dentistry. 2013;41(4):345-55.

34. Zhou H, Liu H, Weir MD, Reynolds MA, Zhang K, $\mathrm{Xu} \mathrm{HH}$. Three-dimensional biofilm properties on dental bonding agent with varying quaternary ammonium charge densities. J Dentistry. 2016;53:73-81.

35. Zhang K, Cheng L, Weir MD, Bai YX, Xu HH. Effects of quaternary ammonium chain length on the antibacterial and remineralizing effects of a calcium phosphate nanocomposite. Int $\mathbf{J}$ Oral Sci. 2016;8(1):45-53.

36. Hegstad K, Langsrud S, Lunestad BT, Scheie AA, Sunde M, Yazdankhah SP. Does the wide use of quaternary ammonium compounds enhance the selection and spread of antimicrobial resistance and thus threaten our health? Microbial drug resistance (Larchmont, NY). 2010;16(2):91-104.

37. Wang S, Wang H, Ren B. Do quaternary ammonium monomers induce drug resistance in cariogenic, endodontic and periodontal bacterial species? Dental Materials. 2017;33(10):1127-38.

38. Imazato S, Torii M, Tsuchitani Y, McCabe JF, Russell RR. Incorporation of bacterial inhibitor into resin composite. J Dent Res. 1994;73(8):1437-43.

39. Sadekuzzaman M, Yang S, Mizan M, Ha S-D. Current and Recent Advanced Strategies for Combating Biofilm. Comprehensive Reviews in Food Science and Food Safety. 2015;14:491-509.

40. Eckert R, Sullivan R, Shi W. Targeted antimicrobial treatment to re-establish a healthy microbial flora for long-term protection. Adv Dent Res. 2012;24(2):94-7.

41. Hogan DA. Talking to themselves: autoregulation and quorum sensing in fungi. Eukaryotic cell. 2006;5(4):613-9.

42. Miller MB, Bassler BL. Quorum sensing in bacteria. Annual Rev Microbiol. 2001;55:165-99.

43. El-Qushayri AE, Dahy A, Reda A. A closer look at the high burden of psychiatric disorders among healthcare workers in Egypt during the COVID-19 pandemic. Epidemiol Health. 2021;43:e2021045.

44. Dibas M, Doheim MF, Ghozy S, Ros MH, El-Helw GO, Reda A. Incidence and survival rates and trends of skull Base chondrosarcoma: A Population-Based study. Clin Neurol Neurosurg. 2020;198:106153.

Cite this article as: Bamofleh SA, Almuammar MS, Osseil AIB, Alorf RA, Alqhtani SM, Alsadah AA, et al. Biological and chemical forms in controlling oral biofilms. Int J Community Med Public Health 2022;9:1123-7. 